\title{
Emphysematous Pyelonephritis with Extensive Myonecrosis: A Case Report
}

\author{
Authors \\ Rajesh G. Halarnakar, Prashant T.N. Mandrekar, Pankaj D. Chari, Veku A. Gaude, \\ Maria Amanda L. Cardoso, Nilesh B. Talwadker, Prashant R. Lawande, \\ Madhumohan R. Prabhudessai \\ Department of Urology, Goa Medical College, Goa-India
}

\begin{abstract}
Emphysematous pyelonephritis (EPN) is a urologic emergency characterized by an acute necrotizing parenchymal and perirenal infection caused by gas-forming uropathogens. This is a case report of a 60 year old female who presented to Goa Medical College with lower abdominal pain, vomiting and inability to walk. On CT scan patient was diagnosed to have bilateral emphysematous pyelonephritis with extension of air into posterior abdominal wall, right gluteal region and right thigh. On examination patient had tenderness in both lumbar and suprapubic region with crepitation over right lower abdomen, back, right gluteal region and right thigh. CT scan revealed bilateral emphysematous pyelonephritis with extension of air into the pelvis, posterior abdominal wall, right gluteal region and right thigh. Blood tests showed leucocytosis with thrombocytopenia with arterial blood gas analysis showing metabolic acidosis. Emergency percutaneous nephrostomy was done bilaterally. Patient subsequently underwent cystocopy with Right DJ Stenting with Left nephrectomy with desloughing and release incision of right buttock, back \& right thigh. Bilateral EPN with extensive myonecrosis is a rare and extremely serious, life threatening condition requiring aggressive management. Aggressive management is needed in the form of wide desloughing and nephrectomy.
\end{abstract}

Keywords: Emphysematous pyelonephritis, myonecrosis, nephrectomy.

\section{Introduction}

Emphysematous pyelonephritis was described in 1898 by Kelly and MacCallum ${ }^{1}$. Emphysematous pyelonephritis (EPN) is a urologic emergency characterized by an acute necrotizing parenchymal and perirenal infection caused by gas-forming uropathogens. Surgical management not only depends on the class of EPN but also on general condition of the patient. The term Emphysematous Pyelonephritis was first used by Schultz and Klorfein. $^{2}$

\section{Case Report}

This is a case report of a 60-year-old female who presented to Goa Medical College with lower abdominal pain, vomiting, and inability to walk. Patient was diabetic on irregular treatment.

On examination, patient was conscious with cold and clammy extremities. Pulse rate was 130 beats/minute and her BP was $80 \mathrm{mmHg}$ systolic. Her respiratory rate was 30 breaths/minute. On examination patient had tenderness in bilateral lumbar and suprapubic region with crepitation over right lower abdomen, back, right gluteal region and right thigh. 
Necrotic patch of skin was present over gluteal region and thigh. CT scan revealed bilateral emphysematous pyelonephritis (Class IV), with

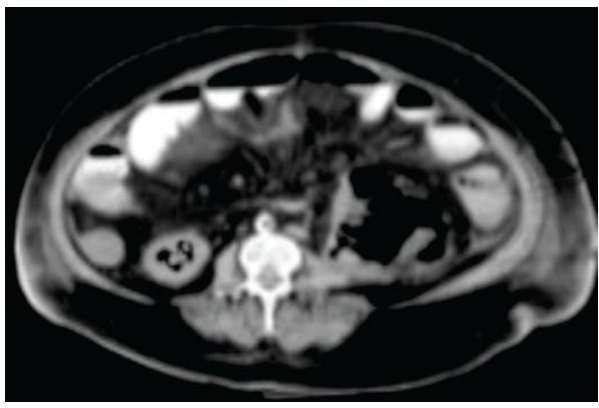

Fig. 1 (A) extension of air into posterior abdominal wall, right gluteal region, and right thigh (Fig. 1, 2 \& 3).

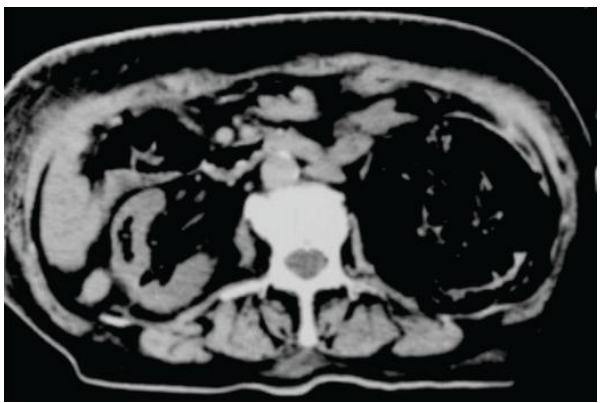

Fig. 1(B)

Fig. 1 (A) \& Fig. 1(B)- Bilateral EPN with Left side kidney showing complete distortion of normal architecture

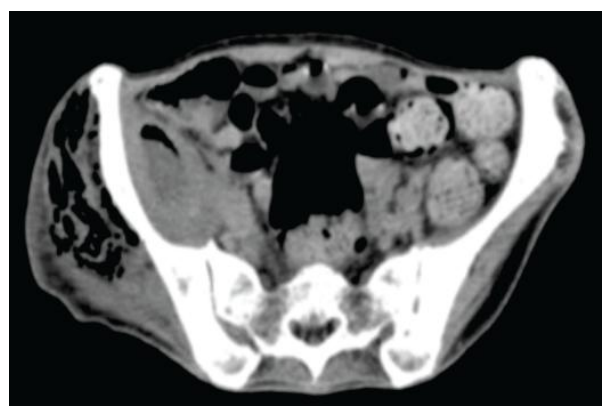

Fig. 2 (A)

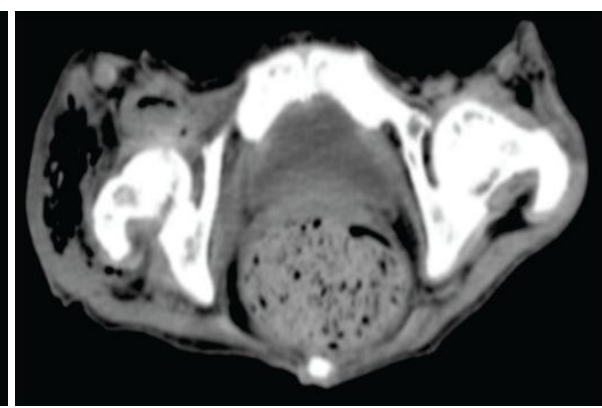

Fig. 2 (B)

Fig. 2 (A) \& Fig. 2(B) - Air in the right gluteal and thigh muscles.

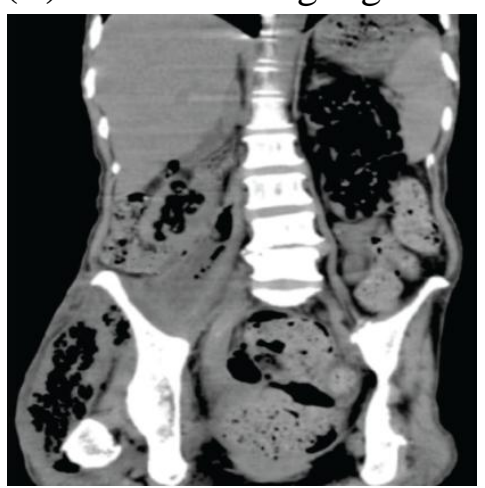

Fig. 3 - CT sagital section showing bilateral EPN with air in right gluteal and thigh muscles

Laboratory investigations were done as shown in Table 1, which revealed leucocytosis with thrombocytopenia with arterial blood gas analysis showing metabolic acidosis.

Patient was resuscitated with IV fluids \& broad spectrum antibiotics, and correction of acid base balance and blood sugars was also done. Emergency percutaneous nephrostomy was done bilaterally.
Patient subsequently underwent cystocopy with Right DJ Stenting with Left nephrectomy with desloughing and release incision of right buttock, back \& right thigh. There was an extensive myonecrosis with collection of pus in the posterior abdominal wall tracking into the gluteal region and thigh. Post- operatively patient was managed in ICU and gradually weaned off the ventilator on 2nd post- op day. Patient was discharged on 15 th post- op day. 
Table 1 Laboratory investigations

\begin{tabular}{|l|c|}
\hline Investigations & Results \\
\hline Haemoglobin & $10.4 \mathrm{gm} \%$ \\
\hline Total count & 22000 (Neutrophil $-84 \%$, Lymphocyte $-16 \%)$ \\
\hline Platelet count & Normal \\
\hline Bleeding Time \& Clotting Time & $424 \mathrm{mg} \%$ \\
\hline RBSL & $2.2 \mathrm{mg} \%$ \\
\hline Sr. Creatinine & Numerous Pus cells \\
\hline Urine Routine & Ketones +, Sugars +++ \\
& Growth of E.coli \& Streptococcus \\
\hline Urine Culture & Sterile \\
\hline Blood Culture & Gram negative rods with gram positive cocci \\
\hline Gram Staining & Metabolic acidosis \\
\hline ABG & \\
\hline
\end{tabular}

\section{Discussion}

Emphysematous pyelonephritis is an acute necrotising parenchymal \& perirenal infection caused by gas forming organisms. It commonly occurs in diabetic patients. E. coli accounts for approximately $70 \%$ of all possible infectious etiologies in $\mathrm{EPN}^{3-5}$. Other organisms are klebsiella, proteus, pseudomonas, streptococci, enterobacter. Many patients have an obstructed system either due to calculi or papillary necrosis. Factors responsible for pathogenesis of EPN are presence of gas forming bacteria, high tissue glucose levels, defective immune response and impaired tissue perfusion ${ }^{6}$. EPN is known to track into ilio- psoas, IVC \& mediastinum. However, extension into lower limbs is rare. The combined occurrence of the necrotising fasciitis with emphysematous pyelonephritis is extremely unusual. Although necrotizing fasciitis can be caused by a retroperitoneal infection, only 3 cases of necrotizing fasciitis associated with emphysematous pyelonephritis have been described previously in the literature ${ }^{7-10}$. Emphysematous pyelonephritis with extensive myonecrosis of gluteus and thigh muscles is extremely rare and only few cases have been reported in literature. Extension of infection from emphysematous pyelonephritis to the adjacent subcutaneous tissue has been proposed ${ }^{8}$. Due to the absence of external muscular layer, the superior and inferior lumbar triangles are two sites of anatomical weakness in the abdominal wall in the flank. The superior lumbar triangle is bounded by the internal oblique muscle anteriorly, the quadratus lumborum muscle posteriorly, and 12th rib superiorly. The inferior triangle is bounded by the external oblique muscle anteriorly, the latissimus dorsi muscle posteriorly, and the iliac crest inferiorly. Through this triangle, necrotizing fasciitis in flank can occur from emphysematous pyelonephritis ${ }^{11}$. Treatment of necrotizing fasciitis is by a combination of appropriate antibiotics, optimal oxygenation of infected tissue, and surgical debridement if necessary ${ }^{10}$.

\section{Conclusions}

Bilateral EPN with extensive myonecrosis is a rare and extremely serious, life threatening condition requiring aggressive management. Aggressive management is needed in the form of wide desloughing and nephrectomy.

\section{References}

1. Kelly HA, MacCallum WG. Pneumaturia. JAMA 1898; 31 : 375 - 81 .

2. Schultz EH Jr, Klorfein EH. Emphysematous Pyelonephritis. J Urol 1962;87:762- 6

3. Chen MT, Huang CN, Chou YH, Huang $\mathrm{CH}$, Chiang $\mathrm{CP}$, Liu GC: Percutaneous drainage in the treatment of emphysematous pyelonephritis: 10-year experience. J Urol 1997; 157:1569-1573.

4. Huang JJ, Tseng CC: Emphysematous pyelonephritis: clinicoradiological classification, management, prognosis, and 
pathogenesis. Arch Intern Med 2000; 160: 797-805.

5. Wan YL, Lee TY, Bullard MJ, Tsai CC: Acute gas-producing bacterial renal infection: correlation between imaging findings and clinical outcome. Radiology 1996; 198: 433-438.

6. Chen KW, H uang JJ, Wu MH, e tal. G as in hepatic veins: a rare and critical presentation of emphysematous pyelonephritis . J Urol 1994 ; 151 : 125 6.

7. Lin DC, Shieh GS, Tong YC. Bilateral emphysematous pyelonephritis combined with subcapsular hematoma and disseminated necrotizing fasciitis. Urol Int 2003;70:244-6.

8. Ishigami K, Bolton-Smith JA, DeYoung BR, Barloon TJ. Necrotizing fasciitis caused by xanthogranulomatous and emphysematous pyelonephritis: importance of the inferior lumbar triangle pathway. AJR Am J Roentgenol 2004;183: 1708-10.

9. Amitai A, Sinert R. Necrotizing fasciitis as the clinical presentation of a retroperitoneal abscess. J Emerg Med 2008;34:37-40.

10. Yu-Xiong Ye, Yao-Ko Wen. Emphysematous Pyelonephritis Presenting as Necrotizing Fasciitis of the Leg. J Chin Med Assoc • March 2009 • Vol 72 • No 3

11. Yasuda T, Tani Y. Necrotizing fasciitis caused by emphysematous pyelonephritis through iliopsoas abscess. J Orthop Sci 2011; 16:832-5. 